\title{
Coupling of surface plasmons between two silver films in a plasmonic thermal emitter
}

\author{
Chia-Yi Chen, Ming-Wei Tsai, Yu-Wei Jiang, Yi-Han Ye, \\ Yi-Tsung Chang, and Si-Chen Lee ${ }^{\text {a) }}$ \\ Department of Electrical Engineering, Graduate Institute of Electronics Engineering, National Taiwan \\ University, Taipei, 10617 Taiwan, Republic of China
}

(Received 31 August 2007; accepted 21 November 2007; published online 13 December 2007)

\begin{abstract}
The emission spectra of $\mathrm{Ag} / \mathrm{SiO}_{2} / \mathrm{Ag}$ trilayer plasmonic thermal emitters with various $\mathrm{SiO}_{2}$ thicknesses were investigated. By analyzing the relationship between emission peaks and thicknesses of $\mathrm{SiO}_{2}$, the coupling of surface plasmons between two silver films in a plasmonic thermal emitter is demonstrated and the coupling length is determined as well. Furthermore, the dispersion relation of plasmonic thermal emitter is detected by measuring the reflection spectra with various incident angles. This confirms that the main mechanism involved in the emission of a plasmonic thermal emitter is due to the excitation of surface plasmons. (c) 2007 American Institute of Physics. [DOI: 10.1063/1.2824389]
\end{abstract}

Surface plasmons (SPs) have been studied for years because of their potential applications in biosensor, photolithography, ${ }^{1,2}$ and light-emitting device. ${ }^{3-6}$ By using thermal radiation and the coupling of surface plasmons with light in periodic metal structure, midinfrared narrowbandwidth plasmonic thermal emitter was demonstrated by Tsai et al. ${ }^{3}$ Theoretically, surface plasmons are induced at the interface between the top patterned silver film and neighboring dielectric materials; thus, the emission peak position can be predicted from the momentum conservation equation and the dispersion relation of surface plasmons. The peak position, however, will deviate from the theoretical value when the thickness of $\mathrm{SiO}_{2}$ is finite. It is because the surface plasmons induced at top and bottom $\mathrm{Ag} / \mathrm{SiO}_{2}$ interfaces are coupled when the $\mathrm{SiO}_{2}$ layer is not thick enough. ${ }^{7}$

In this work, the thermal radiation spectra and reflection dispersion relation of $\mathrm{Ag} / \mathrm{SiO}_{2} / \mathrm{Ag}$ trilayer plasmonic thermal emitters with different $\mathrm{SiO}_{2}$ thicknesses are investigated. By analyzing the shift of peak position, the coupling length of surface plasmons between top and bottom $\mathrm{Ag} / \mathrm{SiO}_{2}$ interfaces is deduced.

The $300 \mathrm{~nm}$ Mo film was deposited by sputtering on the back of the double-polished Si substrate as heating source. $20 \mathrm{~nm}$ Ti and $200 \mathrm{~nm} \mathrm{Ag} \mathrm{metal} \mathrm{films} \mathrm{were} \mathrm{deposited} \mathrm{on} \mathrm{the}$ front side of the $\mathrm{Si}$ substrate followed by a $\mathrm{SiO}_{2}$ layer deposited by electron beam evaporation. Then, a negative photoresist was spun on the $\mathrm{SiO}_{2}$ layer and a hexagonal rod array with the lattice constant $a$ of $3 \mu \mathrm{m}$ and diameter $d$ of $1.5 \mu \mathrm{m}$ was formed on photoresist by photolithography. Finally, a 100-nm-thick silver film was deposited on the photoresist rod array and lifted off to form a silver hexagonal hole array on $\mathrm{SiO}_{2}$ layer. The side and top views of the device are shown in Figs. 1(a) and 1(b), respectively. The devices were heated by sending electric current through the back Mo metal on $\mathrm{Si}$ substrate. A thermal couple is put on the top of the sample to measure the temperature. The thermal radiation generated in the $\mathrm{SiO}_{2}$ layer resonates between the two metal films and the $\mathrm{Ag} / \mathrm{SiO}_{2}$ and $\mathrm{Ag}$ /air surface plasmon polaritons are induced and then converted to light radiation. A

\footnotetext{
${ }^{a)}$ Electronic mail: sclee@cc.ee.ntu.edu.tw.
}

PerkinElmer 2000 Fourier transform infrared spectrometer system was adopted to measure thermal radiation spectra. The wave number resolution of the measurement was $8 \mathrm{~cm}^{-1}$. Furthermore, by measuring the reflection spectra with incident angles of light from $12^{\circ}$ to $65^{\circ}$, the dispersion relations of plasmons in this emitter can be measured clearly. The dispersion relations are measured along the $\Gamma K$ direction, where the position of $K$ in the reciprocal space is shown in Fig. 2.

Figures 3(a)-3(c) show the thermal radiation spectra of emitters at $280{ }^{\circ} \mathrm{C}$ with $\mathrm{SiO}_{2}$ thicknesses of 500, 100, and $60 \mathrm{~nm}$, respectively. In Fig. 3(a), peaks at $3.8 \mu \mathrm{m}$ are due to the degenerate modes composed of $( \pm 1,0) \mathrm{Ag} / \mathrm{SiO}_{2},(0, \pm 1)$ $\mathrm{Ag} / \mathrm{SiO}_{2},(-1,1) \mathrm{Ag} / \mathrm{SiO}_{2}$, and $(1,-1) \mathrm{Ag} / \mathrm{SiO}_{2}$ modes, ${ }^{3}$ designated as $(1,0)$ degenerated $\mathrm{Ag} / \mathrm{SiO}_{2}$ mode. It is almost the same as the theoretical peak position at $3.79 \mu \mathrm{m}$. The peak located at $3 \mu \mathrm{m}$ is due to the cross coupling of the

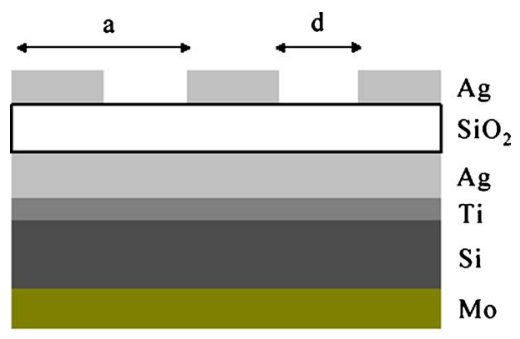

(a)

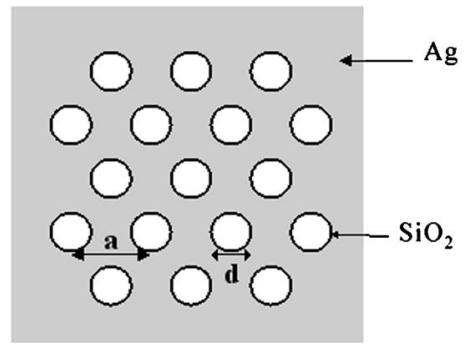

(b)

FIG. 1. (Color online) Plasmonic infrared emitter structure, (a) side and (b) top views. 


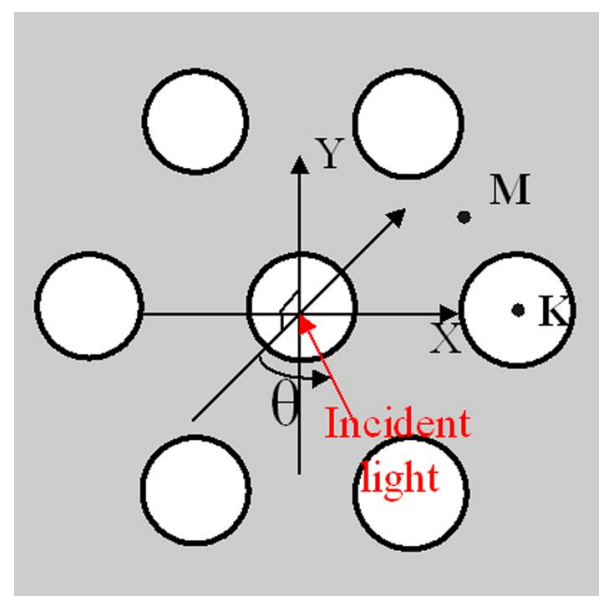

FIG. 2. (Color online) The definition of $\Gamma K$ direction with respect to the surface structure geometry in reciprocal lattice.

$\mathrm{Ag} / \mathrm{SiO}_{2}$ mode and $\mathrm{Ag} /$ air mode. ${ }^{3}$ When the thickness of $\mathrm{SiO}_{2}$ reduces from 500 to $100 \mathrm{~nm}$, the emission peak shifts from 3.8 to $4.56 \mu \mathrm{m}$, as shown in Fig. 3(b). As the thickness of $\mathrm{SiO}_{2}$ becomes $60 \mathrm{~nm}$, the emission peak shifts further to $5.34 \mu \mathrm{m}$, as shown in Fig. 3(c), which is far away from the theoretical value. More light is generated in the thicker $\mathrm{SiO}_{2}$ layer and the degenerate $(1,0) \mathrm{Ag} / \mathrm{SiO}_{2}$ modes are easier to distinguish. The redshift of emission peaks with the reduction of $\mathrm{SiO}_{2}$ thickness results from the coupling of surface plasmons between top and bottom metal plates. It is because the surface plasmons are also induced at the interface between the bottom silver film and $\mathrm{SiO}_{2}$ by the field of surface plasmons in top silver film when the thickness of $\mathrm{SiO}_{2}$ is not thick enough.

Figures 4(a)-4(d) display the reflection dispersion relations of emitters with $\mathrm{SiO}_{2}$ thicknesses of 500, 100, 60, and $0 \mathrm{~nm}$, respectively, for light propagating in $\Gamma K$ direction with different incident angles. Dark lines on dispersion relation represent the dips in reflection spectra. Light which satisfies the momentum conservation and the dispersion relation of surface plasmons can couple with the surface plasmons.

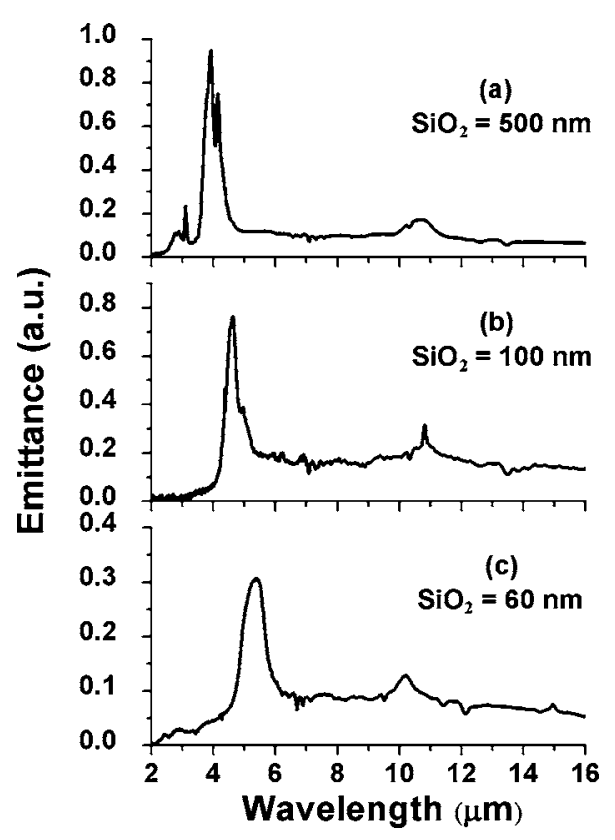

FIG. 3. Measured emission spectra of emitters with $a=3 \mu \mathrm{m}$ and $d$ $=1.5 \mu \mathrm{m}$, and the thicknesses of $\mathrm{SiO}_{2}$ are (a) 500, (b) 100, and (c) $60 \mathrm{~nm}$.

Therefore, less light is reflected from the emitters when satisfied with the coupling conditions. The six degenerate modes at $3.8 \mu \mathrm{m}(0.326 \mathrm{eV})$ for normal incidence light split into four dispersion lines, as denoted in Fig. 4(a), when light incident angle increases (vector $\overrightarrow{\mathbf{k}}_{x}$ increases). This dispersion relation provides evidence that the main mechanism of the thermal emission peak is due to the coupling of light with surface plasmons. Other dark lines in higher energy region are due to the higher order modes of surface plasmons. When the thickness of $\mathrm{SiO}_{2}$ is $100 \mathrm{~nm}$, the dispersion relation is still the same but with all bands shift to lower energy slightly. Figure 4(c) shows the dispersion relation of emitter with $\mathrm{SiO}_{2}$ thickness of $60 \mathrm{~nm}$. Now, the degenerate $\mathrm{Ag} / \mathrm{SiO}_{2}$ modes are at $0.23 \mathrm{eV}$ which is consistent with the radiation emission peak at $5.34 \mu \mathrm{m}(0.23 \mathrm{eV})$ shown in Fig. 4(c). If

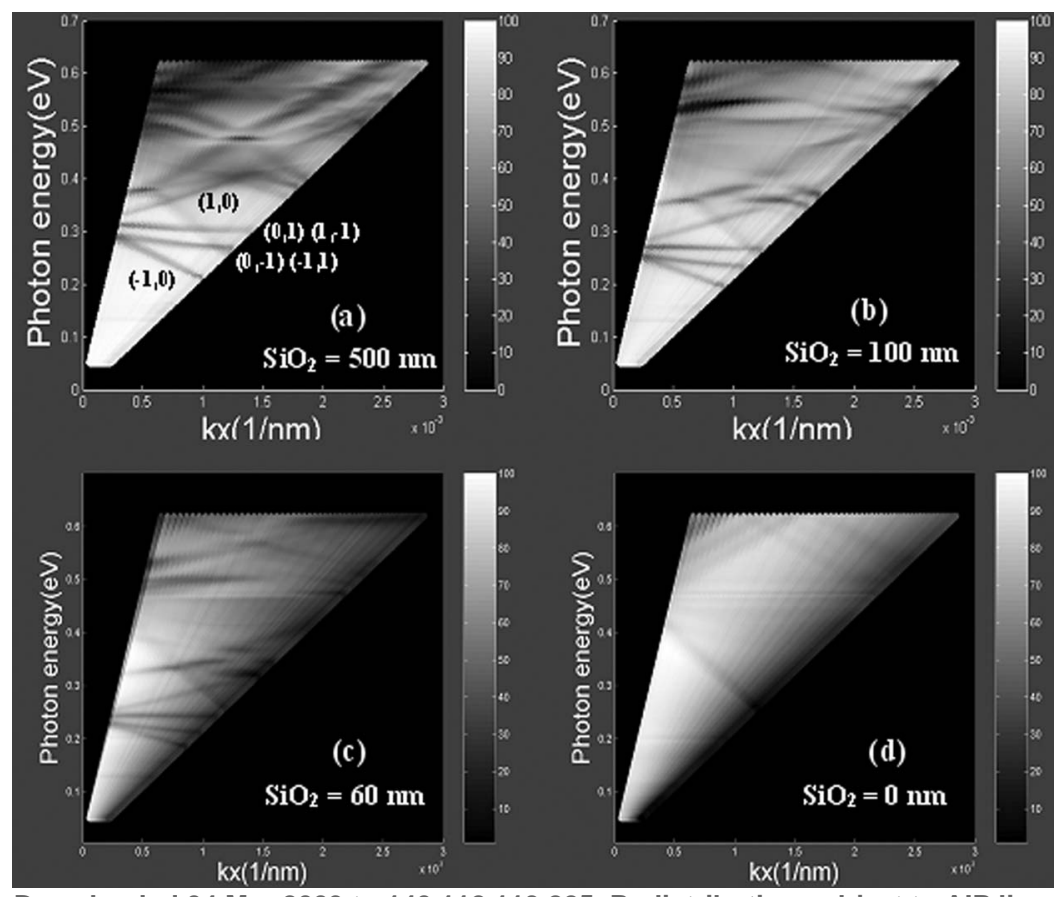

FIG. 4. Energy dispersions of thermal emitters with $a$ $=3 \mu \mathrm{m}$ and $d=1.5 \mu \mathrm{m}$, and the thicknesses of $\mathrm{SiO}_{2}$ are (a) 500, (b) 100 , (c) 60, and (d) $0 \mathrm{~nm}$ for light propagating along the $\Gamma K$ direction. 


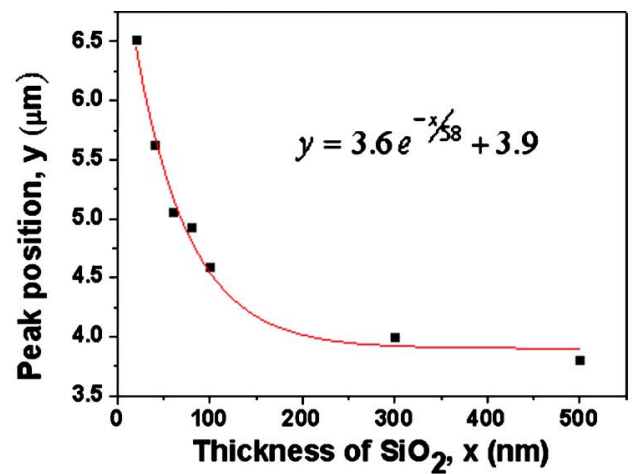

(a)

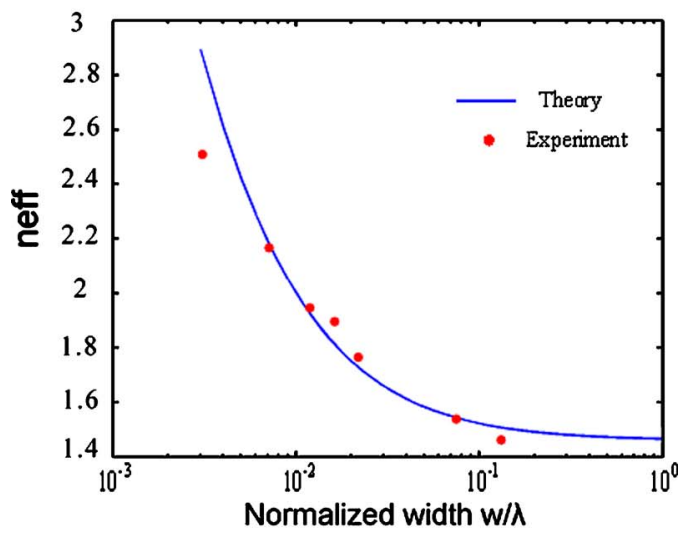

(b)

FIG. 5. (Color online) (a) The relationship between the peak wavelength of $(1,0) \mathrm{Ag} / \mathrm{SiO}_{2}$ mode vs the thickness of $\mathrm{SiO}_{2}$. Square dots are the experimental results and the red line is the fitting curve. (b) Effective refractive index $n_{\text {eff }}$ as a function of normalized $\mathrm{SiO}_{2}$ thickness $w / \lambda$. The blue line is the theoretical curve and red circles are experimental data.

no $\mathrm{SiO}_{2}$ layer exists in the plasmonic emitter, i.e., thickness of $\mathrm{SiO}_{2}$ equals to $0 \mathrm{~nm}$, the dispersion curves due to the $\mathrm{Ag} / \mathrm{SiO}_{2}$ surface plasmon modes all disappear except that of the Ag/air mode, as shown in Fig. 4(d). This confirms again that the physical origin involved in the thermal emitter is the excitation of surface plasmons at the $\mathrm{Ag} / \mathrm{SiO}_{2}$ interface. The split behaviors are all the same for emitters with different $\mathrm{SiO}_{2}$ thicknesses. It means that the coupling of surface plasmons changes the energy of themselves. If the coupling effect is enhanced, surface plasmons can be induced by the light with longer wavelength, even though the periodic structure on top Ag film is the same.

The relation between peak position of degenerate $(1,0)$ $\mathrm{Ag} / \mathrm{SiO}_{2}$ mode and $\mathrm{SiO}_{2}$ is shown in Fig. 5(a). The peak position can be fitted using an exponential function to determine the coupling length of $(1,0) \mathrm{Ag} / \mathrm{SiO}_{2}$ mode in the $\mathrm{Ag} / \mathrm{SiO}_{2} / \mathrm{Ag}$ triple layer structure. The fitting curve is $y$ $=3.6 e^{-x / 58}+3.9$, where $x$ is the thickness of $\mathrm{SiO}_{2}$ in nanometers. As the thickness of $\mathrm{SiO}_{2} x$ increases to infinity, the peak position $y$ approaches $3.9 \mu \mathrm{m}$ which is very close to the theoretical value of $3.79 \mu \mathrm{m}$. The coupling length in this structure is $58 \mathrm{~nm}$ which is much shorter than that of $\mathrm{Ag} / \mathrm{SiO}_{2}$ mode in an $\mathrm{Ag} / \mathrm{SiO}_{2} / \mathrm{Si}$ triple layer structure. ${ }^{8}$ It is due to the strong coupling of surface plasmons between the two silver films. It can be seen that when the thickness of $\mathrm{SiO}_{2}$ is less than $100 \mathrm{~nm}$, the peak position redshifts substantially with the reduction of $\mathrm{SiO}_{2}$ thickness. The coupling of surface plasmons becomes less apparent when the $\mathrm{SiO}_{2}$ thickness exceeds $300 \mathrm{~nm}$.

Collin et $a l .{ }^{7}$ have theoretically calculated the coupling strength of surface plasmons in a metal/dielectric/metal structure. Their conclusion is that the coupling of surface plasmons increases the effective refractive index of the intermediate dielectric medium resulting in the redshift of wavelength. According to their work, the equation of effective refractive index of transverse magnetic mode in a thin dielectric layer sandwiched by two metal layers is given as follows:

$$
n_{\mathrm{eff}}=\frac{k_{x}}{k_{0}}=\sqrt{\varepsilon_{d}}\left(1+\frac{\lambda}{\pi w \sqrt{-\varepsilon_{m}}} \sqrt{1+\frac{\varepsilon_{d}}{-\varepsilon_{m}}}\right)^{1 / 2}
$$

where $w$ is the thickness of intermediate dielectric layer, $\lambda$ is the wavelength in free space, and $\varepsilon_{d}$ and $\varepsilon_{m}$ are the permittivities of dielectric and metal mediums, respectively. By comparing our result to the Eq. (1), the effective refractive index $n_{\text {eff }}$ as a function of normalized width $w / \lambda$ is shown in Fig. 5(b). The blue line is the theoretical curve drawn by Eq. (1) and the red circles are experimental data. With increasing $w / \lambda$, the effective refractive index becomes smaller, finally approach the $\sqrt{\varepsilon_{d}}$ value.

In conclusion, the emission spectra and reflection dispersion relations of $\mathrm{Ag} / \mathrm{SiO}_{2} / \mathrm{Ag}$ thermal plasmonic emitters with different $\mathrm{SiO}_{2}$ thicknesses were investigated experimentally. The position of thermal emission peak of a plasmonic emitter is not only determined by the periodic structure on top Ag film but is also affected by the thickness of intermediate $\mathrm{SiO}_{2}$. Surface plasmons at the top and bottom $\mathrm{Ag} / \mathrm{SiO}_{2}$ interfaces couple together if the thickness of $\mathrm{SiO}_{2}$ is not thick enough. This coupling effect makes the effective refractive index of $\mathrm{SiO}_{2}$ increase and, therefore, results in the redshift of the $\mathrm{Ag} / \mathrm{SiO}_{2} \mathrm{SP}$ modes. The coupling length turns out to be $58 \mathrm{~nm}$.

The authors would like to thank the National Science Council of the Republic of China for financial support under Coutract No. NSC95-2215-E-002-007.

${ }^{1}$ D. B. Shao and S. C. Chen, Appl. Phys. Lett. 86, 253107 (2005).

${ }^{2}$ X. Luo and T. Ishihara, Appl. Phys. Lett. 84, 4780 (2004).

${ }^{3}$ M.-W. Tsai, T.-H. Chuang, C.-Y. Meng, Y.-T. Chang, and S.-C. Lee, Appl. Phys. Lett. 89, 173116 (2006).

${ }^{4}$ S. Wedge, J. A. E. Wasey, W. L. Barnes, and I. Sage, Appl. Phys. Lett. 85, 182 (2004)

${ }^{5}$ H. L. Tam, K. F. Li, K. W. Cheah, J. B. Xia, R. Huber, W. H. Wong, and Y. B. Pun, Appl. Phys. Lett. 89, 131123 (2006).

${ }^{6}$ S. A. Kalele, N. R. Tiwari, S. W. Gosavi, and S. K. Kulkarni, Journal of Nanophotonics 1, 012501 (2007).

${ }^{7}$ S. Collin, F. Pardo, and J.-L. Pelouard, Opt. Express 15, 4310 (2007).

${ }^{8}$ T.-H. Chuang, M.-W. Tsai, Y.-T. Chang, and S.-C. Lee, Appl. Phys. Lett. 89, 033120 (2006). 